\title{
Publisher's Note: Security of Device-Independent Quantum Key Distribution in the Bounded-Quantum-Storage Model [Phys. Rev. X 3, 031007 (2013)]
}

S. Pironio, Ll. Masanes, A. Leverrier, and A. Acín

(Received 17 January 2014; published 31 January 2014)

DOI: 10.1103/PhysRevX.4.019901

Subject Areas: Quantum Physics, Quantum Information

This paper was published online on 9 August 2013 with partial affiliation information for the second author and incomplete funding information. Ll. Masanes's affiliation should read ICFO-Institut de Ciencies Fotoniques, 08860 Castelldefels, Barcelona, Spain, and H. H. Wills Physics Laboratory, University of Bristol, Tyndall Avenue, Bristol BS8 1TL, United Kingdom. The following sentence is to be added at the end of the Acknowledgment section: "Financial support was also provided by EU ERC Advanced Grant No. NLST (PHYS RQ8784), the EU Qessence Project, and the Templeton Foundation." 